\title{
Redução de preço de medicamento em situação de monopólio no Sistema Único de Saúde: o caso do Tenofovir
}

\section{| ${ }^{1}$ Gabriela Costa Chaves, ${ }^{2}$ Lia Hasenclever, ${ }^{3}$ Maria Auxiliadora Oliveira |}

Resumo: O objetivo do estudo é analisar a evolução do preço do tenofovir (TDF) no Brasil à luz das diferentes iniciativas para sua redução. Os critérios para a seleção do caso foram: ter sido objeto de pelo menos uma estratégia de enfrentamento da barreira patentária, que no caso foi o subsídio ao exame do pedido da patente (oposição à patente); e ter sido objeto de uma Parceria para Desenvolvimento Produtivo (PDP) para produção local. Os principais resultados sugerem que os subsídios ao exame apresentados em 2005 e 2006 contribuíram para a decisão de indeferimento do pedido de patente em 2009. Estimase que o Brasil pagou cerca de US\$ 200 milhóes a mais pelo monopólio gerado a partir de um pedido de patente pendente. Houve uma reduçáo do preço do TDF entre 2003 e 2013, inclusive durante a vigência da PDP (2011 a 2013). Em 2010, após o anúncio da PDP, também houve uma diminuiçáo de $40 \%$ no preço do TDF ofertado pela Gilead, que refletiu no preço de oferta do produto PDP. No entanto, o preço pago no Brasil para o produto nacional foi cerca de dez vezes mais caro que o genérico ofertado internacionalmente.

> Palavras-chave: Preço de antirretrovirais; HIVIAIDS; patentes; medicamentos genéricos; subsídio ao exame.

\author{
'Escola Nacional de Saúde \\ Pública, Fundação Oswaldo \\ Cruz. Rio de Janeiro-RJ, Brasil \\ (gabicostachaves@ensp.fiocruz.br). \\ ORCID: 0000-0002-8347-6164 \\ 2 Instituto de Economia, \\ Universidade Federal do Rio de \\ Janeiro. Rio de Janeiro-RJ, Brasil \\ (lia@ie.ufrj.br). \\ ORCID: 0000-0003-1384-6323 \\ ${ }^{3}$ Escola Nacional de Saúde \\ Pública, Fundação Oswaldo \\ Cruz. Rio de Janeiro-RJ, Brasil \\ (dorabermudez@gmail.com). \\ ORCID: 0000-0003-2400-536X
}

Recebido em: 14/07/2017 Aprovado em: 28/10/2017 Revisado em: 17/12/2017 


\section{Introdução}

O Sistema Único de Saúde (SUS) prevê, entre seus campos de atuação, a execução de açôes de assistência terapêutica integral, incluindo a farmacêutica (BRASIL, 1990), refletida em políticas (BRASIL, 1998; BRASIL, 2004) que possibilitam assegurar a oferta de medicamentos pelo setor público com a finalidade de alcançar o acesso universal. Um dos principais desafios para a sustentabilidade dessas políticas tem sido o aumento das despesas públicas com medicamentos (VIEIRA, 2009), muitas vezes relacionadas à incorporação e compra daqueles de alto custo (FONSECA; COSTA, 2015). As despesas do Ministério da Saúde (MS) com a assistência farmacêutica no SUS passaram de R $\$ 1,95$ bilhão em 2003 para $\mathrm{R} \$ 12,4$ bilhôes em 2014 (BRASIL, 2014a).

Alguns medicamentos apresentam características específicas quanto à sua aquisição no mercado brasileiro, podendo ser comprados exclusivamente pelo setor público (monopsônio) (SILVA, 1999). A oferta de medicamentos no País por um único fornecedor, por sua vez, reflete os direitos de proteção patentária (patente concedida no País) ou a expectativa de direitos (pedido de patente depositado ainda em análise), configurando assim uma situação de monopólio (CHAVES et al., 2015).

Os medicamentos antirretrovirais (ARV), utilizados para o controle da infecção pelo HIV, são um exemplo de produtos comprados exclusivamente pelo setor público e alguns deles encontram-se em situação de monopólio por estarem sujeitos à proteção patentária (CHAVES et al., 2015). Isso porque, a partir de 1995, entrou em vigor o Acordo Trips ${ }^{1}$ da Organização Mundial do Comércio (OMC), o qual estabelece aos seus países membros a obrigatoriedade de reconhecimento de patentes para todos os campos tecnológicos, incluindo o setor farmacêutico.

No Brasil, o Acordo Trips foi internalizado na lei de propriedade industrial $n^{\circ}$ 9.279/96, passando a assegurar proteção de patentes no setor farmacêutico a partir de maio de 1997 e também por um mecanismo conhecido como pipeline (OLIVEIRA et al., 2004; HASENCLEVER et al., 2010).

Experiências internacionais de regulação de preços de medicamentos patenteados incluem instrumentos como controle direto de preços por meio da utilizaçáo de preços de referências internacionais, negociação de preços ou preços de referência baseados em produtos de uma mesma classe terapêutica (SOOD et al., 2009). 
Os ARV são importantes objetos para a análise dos desafios governamentais em reduzir preços de medicamentos em situação de monopólio. Eles foram os primeiros produtos patenteados incorporados no SUS, inserindo na agenda governamental o desafio da sustentabilidade da oferta do tratamento de produtos de altos preços e alto gasto (GRANGEIRO et al., 2006; NUNN et al., 2007). Também são exemplares na adoção de estratégias para redução de preços, o que se reflete num histórico recente de manutenção das despesas com aumento do número de pessoas em tratamento (FONSECA; COSTA, 2015).

Além dos mecanismos de regulação de preços no momento da entrada do produto no País e dos ajustes anuais estabelecidos pela Câmara de Regulação do Mercado de Medicamentos (CMED) (ANVISA, 2004), também é possível alcançar reduções no processo das compras públicas.

O governo brasileiro tem empreendido esforços governamentais para a redução de preços dos ARV em situação de monopólio, que, em alguns casos, incluíram uma combinação de estratégias que possibilitaram aumentar o poder de barganha do MS no processo de negociação de preços com empresas multinacionais, quais sejam: não reconhecimento dos direitos de propriedade industrial para o setor farmacêutico, no período pré-Trips; uso da produção pública de medicamentos para estimar o custo de produção dos produtos, apresentando preços de referência e desenvolvimento desses produtos; e enfrentamento da barreira patentária, por meio da ameaça e/ou uso de licenciamento compulsório e apresentação de subsídios ao exame, no período pós-Trips (CHAVES et al., 2015).

O tenofovir (TDF) é um ARV da classe dos inibidores da transcriptase reversa análogos de nucleotídios (ITRN), que foi aprovado para indicação de uso em HIV nos Estados Unidos em outubro de 2001 por solicitação da empresa Gilead. A aprovação no Brasil pela Anvisa ocorreu 19,6 meses após a aprovação pelo FDA (SCHEFFER, 2005; SCHEFFER et al., 2008). Sua incorporação no elenco de ARV oferecidos pelo SUS se deu em 2003, sendo recomendado no consenso de 2004 como uma das alternativas à zidovudina (AZT), utilizada no esquema de primeira linha. No consenso de 2008, o TDF passou a ser alternativa preferencial ao AZT no esquema de primeira linha e também uma das opçóes dos esquemas de segunda linha (MEINERS, 2012). Em 2005, a Gilead foi uma das três empresas-alvo do Ministério da Saúde (MS) no processo de negociação de preço, já que os gastos do MS com três produtos em situação de monopólio no país, TDF, lopinavir/ritonavir 
(Lop/r) e efavirenz (EFV), consumiam 80\% do orçamento para compra de ARV (CONSELHO NACIONAL DE SAÚDE, 2005).

O objetivo do presente estudo é analisar a evolução do preço do TDF no Brasil à luz das diferentes iniciativas para sua redução. Estudos anteriores buscaram mapear iniciativas de enfrentamento da barreira patentária de ARV patenteados e relacionar com a evolução do preço do medicamento no Brasil (CHAVES et al., 2015; SCOPEL; CHAVES, 2016). O presente estudo soma-se aos esforços desses estudos, buscando analisar um caso no qual a situação de monopólio se deu por um pedido de patente pendente.

Iniciativas de enfrentamento dessa barreira patentária são aquelas que visam diminuir a situação de monopólio decorrente do pedido de patente ou patente concedida. Segundo as autoras, "mesmo que essas iniciativas não reflitam indeferimento, revogação ou licenciamento da patente, elas podem ter efeito sobre a dinâmica do preço do medicamento" (SCOPEL; CHAVES, 2016; p. 2).

Dois pressupostos norteiam a análise. O primeiro considera que a regulação de preços de medicamentos em situação de monopólio, que forem adotados pelo SUS, não deve se restringir apenas à adoçáo de preços de referência ou escala (volume), mas requer um conjunto de estratégias que envolvam o enfrentamento da barreira patentária, monitoramento da dinâmica internacional do mercado do produto e iniciativas de produção local. O segundo considera que, em alguns momentos, há limites na adoção simultânea de estratégias que envolvam regulação de preços e desenvolvimento industrial nacional.

\section{Metodologia}

Trata-se de estudo de caso envolvendo o ARV fumarato de tenofovir desoproxila (TDF), incorporado no SUS em 2003. Os critérios para a seleção do caso foram: ter sido objeto de pelo menos uma estratégia de enfrentamento da barreira patentária, que no caso foi o subsídio ao exame do pedido da patente, e ter sido objeto de uma Parceria para Desenvolvimento Produtivo (PDP) para produção local.

O mapeamento das iniciativas de enfrentamento da barreira patentária no Brasil foi realizado mediante levantamento da literatura (FLYNN, 2010; BARROSO, 2013; VERAS, 2014; CHAVES et al., 2008; REIS et al., 2009; BARROSO, 2010), análise do andamento de pedidos de patente do TDF no endereço eletrônico 
do Instituto Nacional de Propriedade Industrial (INPI); análise da legislação de propriedade industrial; e entrevistas com dois informantes-chave do Ministério da Saúde no período analisado.

A fatia do mercado brasileiro de TDF diante das vendas totais do medicamento pela empresa Gilead correspondeu aos anos de 2003 a 2010, período em que a empresa o forneceu ao governo brasileiro. Os gastos com TDF no período foram estimados a partir do preço de aquisição e volume pelo Ministério da Saúde obtidos pela Lei de Acesso à informação. Os preços foram convertidos para dólares americanos (US\$) pelo dólar médio do ano (IPEA-data). Os valores de vendas mundiais foram obtidos pela publicação de Médicos Sem Fronteiras, intitulada Untangling the Web of ARV Price reduction (ACCESS CAMPAIGN/MSF, 2014).

A análise da produção local do TDF teve como fonte de dados: cópia do acordo de uma das PDP para o TDF, firmado entre o parceiro público e parceiros privados, cedido pela Associação Brasileira Interdisciplinar de Aids (ABIA); entrevistas com dois representantes do Laboratório Farmacêutico Oficial (LFO) envolvido; e um dos parceiros privados, mediante aplicação de roteiro semiestruturado. A análise centrouse nos aspectos relacionados à redução do preço do produto no contexto da PDP.

Para análise da evolução do preço praticado no Brasil, foram utilizados dados obtidos junto ao Ministério da Saúde por meio da Lei de Acesso à Informação para os anos de 2003 a 2013, sendo este recorte definido pelo ano da incorporação do medicamento no SUS. Os preços em reais $(\mathrm{R} \$)$ foram corrigidos pela inflação, medida pelo IPCA de 2013. O volume adquirido por ano foi expresso em números de tratamentos estimados, baseado na posologia de 1 comprimido por dia/ano² (MEINERS, 2012).

Para fins de comparação do preço com a versão genérica indiana, optou-se por utilizar os preços praticados pela empresa Cipla, a partir de 2006. A opção deveu-se ao fato de esta ter sido a única empresa indiana que teria condiçóes legais de exportar para o Brasil, pois as demais empresas estavam impossibilitadas de exportar para o Brasil por terem firmado acordos de licença voluntária com a Gilead, cujo escopo geográfico de comercialização excluía o Brasil (ACCESS CAMPAIGN/MEDECINS SANS FRONTIÈRES, 2013). A estimativa do quanto o governo brasileiro pagou a mais pelo medicamento durante o período de monopólio envolvendo o pedido de patente pendente foi baseada na diferença entre os preços praticados pela Gilead e aqueles praticados pela Cipla multiplicados pela quantidade do produto adquirido pelo Ministério da Saúde no período de 2006 a 2010. 
O presente estudo foi aprovado pelo Comitê de Ética em Pesquisa da ENSP/ Fiocruz, em 8 de agosto de 2013, em parecer consubstanciado no 353.233 (CAAE 19480113.6.0000.5240).

\section{Resultados e Discussão}

\section{Iniciativas de enfrentamento da barreira patentária e a dinâmica do mercado internacional do TDF}

Em 2005, a situação de monopólio do TDF se caracterizava de duas formas. Primeiro, por não haver até aquele momento nenhuma versão genérica do medicamento disponível no mercado internacional. Segundo, porque os pedidos de patente depositados no Brasil estavam pendentes de decisão (em análise). Ressalta-se como pedido mais importante para a empresa o PI9811045-4, referente ao sal de fumarato do pró-fármaco disoproxil tenofovir (REIS, 2012). A simples expectativa de direito decorrente de pedidos de patente ainda pendentes de decisão no INPI configurou por si só uma situação de monopólio mesmo não existindo patente concedida.

A Lei no 9.279/96, sobre os direitos de propriedade industrial, assegura o direito de o titular da patente obter indenização relativa à sua exploração indevida mesmo antes de sua concessão (artigo 44). A situação de monopólio decorrente de pedidos de patente pendentes pode ser resultante de uma interpretação desse artigo, gerando incerteza jurídica quanto à possibilidade de entrada de outros concorrentes no mercado até a definição da patentabilidade do produto.

O fato de o MS ser o único comprador de ARV no país coloca a empresa detentora do medicamento de referência em vantagem para estabelecer preço no período em que houver incerteza sobre a patenteabilidade do produto. Esta incerteza se fortalece ainda mais em função da prática do evergreening, na qual a empresa solicita vários pedidos de patente relacionados a um mesmo medicamento ou com pequenas modificaçóes com vistas a estender o monopólio do produto (KAPCZYNSKI et al., 2012). Isso contribui para um acúmulo de pedidos de patentes no INPI incompatível com a capacidade de análise pelo órgão, constituindo, portanto, o backlog de patentes (ABREU, 2017).

Embora legalmente o MS tenha a opção de comprar o produto enquanto os pedidos de patente estiverem pendentes de decisão, esta prática não parece ter acontecido no presente caso. Provavelmente não houve a intenção de correr o risco de fazer uma compra diante a possibilidade de uma concessão futura da patente. 
Nos meses de dezembro de 2005 e de 2006 foram apresentados subsídios ao exame contestando um dos pedidos de patente (PI9811045-4) depositados pela Gilead no Brasil. O primeiro foi apresentado por Farmanguinhos/Fiocruz(BARROSO, 2010; 2013) e o segundo, por organizações membros do Grupo de Trabalho sobre Propriedade Intelectual da Rede Brasileira pela Integração dos Povos (GTPI/Rebrip) (REIS et al., 2009). A argumentação principal foi a falta de atividade inventiva (VERAS, 2014). Em janeiro de 2007, Farmanguinhos/Fiocruz apresentou um segundo subsídio ao exame para o mesmo pedido (INPI, 2017).

$\mathrm{Na}$ Índia, empresas privadas nacionais lançaram a versão genérica do TDF em 2006, apesar de os pedidos de patente depositados pela Gilead estarem pendentes naquele país. Provavelmente elas assumiram o risco porque avaliaram que esses pedidos não seriam concedidos. No mesmo ano, a Gilead estabeleceu acordos de licença voluntária com algumas empresas indianas para a produção e comercialização do TDF mediante condições específicas (ACCESS CAMPAIGN/MEDECINS SANS FRONTIÈRES, 2007; NUNN et al., 2007). A única empresa indiana que não fez acordo com a Gilead para produção do TDF foi a Cipla (ACCESS CAMPAIGN/MEDECINS SANS FRONTIÈRES, 2013).

Diante do contexto de apresentação de subsídios ao exame, em 2008 o MS declarou o interesse público do TDF para fins de exame prioritário do pedido de patente PI 9811045 (Portaria no 681/2008) (BRASIL, 2008). A Portaria mencionou o subsídio ao exame apresentado por Farmanguinhos/Fiocruz, a negativa da patente nos Estados Unidos (patente US5.935.946) em janeiro daquele mesmo ano e reconheceu a situação de monopólio num cenário de expectativa de direito ("Considerando que a apresentação de pedido de patente ao INPI gera expectativa de direito monopolístico, com impacto no preço do produto...”).

Este movimento por parte do MS foi possível devido à Resolução do INPI no 132/2006, que possibilitava a solicitaçáo de exame prioritário de um determinado pedido de patente para os casos em que o medicamento fosse declarado de interesse público (artigo $3^{\circ}$ ). Isso permitiu lidar com a questão do backlog do INPI, já que o depósito do pedido era de 1998. O exame prioritário foi concedido no dia 15 de maio de 2008.

O pedido da patente foi indeferido em 26 de agosto de 2008. A Gilead recorreu da decisão, mas o indeferimento do pedido de patente foi mantido e publicado 
no dia 30 de junho de 2009, finalizando assim o processo pela via administrativa (INPI, 2017). Em seguida, a empresa entrou com açáo judicial contestando a decisão final do INPI (VERAS, 2014).

No MS, o Departamento de IST/Aids e Hepatites Virais (doravante IST/Aids/ HV) teve papel relevante no desencadeamento da decisão de declarar o interesse público do TDF para fins de exame prioritário (entrevista gestor público do Ministério da Saúde\#1). Somente após o término do exame do pedido de patente seria possível mobilizar outras iniciativas relacionadas à ampliação do acesso ao TDF, incluindo a produção local.

$\mathrm{O}$ IST/Aids/HV apresentou o cenário e a justificativa para a declaração do interesse público ao então Secretário da Secretaria de Ciência e Tecnologia e Insumos Estratégicos (SCTIE), tendo como base levantamentos do mercado internacional e disponibilidade de alternativas genéricas. $\mathrm{O}$ principal argumento de convencimento foi que a decisão sobre a patenteabilidade poderia contribuir para a política de produção local, sendo, portanto, uma estratégia casada - patente e produção local (entrevista gestor público do Ministério da Saúde\#1). Além da estratégia de solicitar vários pedidos de patente relacionados ao TDF (REIS, 2012), a Gilead também adotou outras táticas visando à preservação do monopólio do medicamento após a decisão pelo indeferimento da patente principal (PI9811045-4) em 2008.

A primeira foi dar entrada com pedido de divisão em 31 de março de 2009 (PI 9816239-0). Esta possibilidade é permitida pela legislaçáo, com a condição de que o pedido dividido esteja relacionado ao pedido original e náo exceda o que está previsto no pedido original (artigo 26, Lei no 9.279/96). O referido pedido foi alvo de um subsídio ao exame técnico apresentado por organizaçóes membros do GTPI/Rebrip no mesmo ano (VERAS, 2014) com o argumento de que a empresa incorporara novas reivindicaçôes ao pedido, inexistentes no pedido original. Embora a patente original tenha sido negada em junho de 2009, o pedido dividido seguiu e foi indeferido em 10 de maio de 2011, tendo sido objeto de recurso pela Gilead em julho do mesmo ano, e com a decisão final pela manutenção do indeferimento publicada em setembro de 2014 (INPI, 2014).

A segunda tática adotada pela empresa foi a utilização da via judicial para contestar a decisão do órgão governamental pelo indeferimento do pedido de patente. Uma vez esgotadas as possibilidades de recursos na via administrativa, a empresa entrou com 
uma "ação ordinária de nulidade de decisão administrativa praticada pelo INPI" em abril de 2010 (VERAS, 2014). Em 2016, o processo foi extinto.

Paralelamente, foram identificados outros movimentos de enfrentamento da barreira patentária no nível internacional. $\mathrm{O}$ primeiro refere-se às oposições a pedidos de patente que, para o TDF, começaram a ser apresentadas a partir de 2006, concomitantes com as iniciativas de subsídio ao exame no Brasil. Veras (2014) caracteriza bem o processo de conexão entre as diferentes iniciativas de oposição das patentes do TDF: a versão apresentada por Farmanguinhos/Fiocruz no Brasil foi compartilhada com MSF e com o Lawyers Collective HIVIAIDS Unit, na Índia, cujo conteúdo contribuiu para validar a oposição que eles estavam elaborando e que foi apresentada em setembro de 2006; a versão elaborada na Índia foi compartilhada com a organização PubPat (Public Patent Foundation) nos Estados Unidos, que, posteriormente, também apresentou oposiçōes para o TDF naquele país (PUBLIC PATENT FOUDANTION, 2007); em 2008, a Associação Brasileira Interdisciplinar de Aids (Abia) e a organizaçáo indiana Sahara apresentaram uma oposiçáo na Índia para um dos pedidos de patente.

Embora tenha levado alguns anos entre a apresentaçáo das oposiçóes e a publicação das decisōes dos escritórios de patentes, o fato é que esses pedidos de patente para o TDF foram sendo sistematicamente negados naqueles países (ACCESS CAMPAIGN/MEDECINS SANS FRONTIÈRES, 2016).

Outra iniciativa refere-se ao pool de patentes, uma abordagem para gestáo da propriedade intelectual que consiste na possibilidade de diferentes instituiçóes, que detêm direitos patentários, colocarem suas patentes num pool para que possa ser gerido e licenciado para outras instituiçôes e empresas. Trata-se de um mecanismo voluntário, que depende que os detentores dos direitos aceitem que suas patentes sejam gerenciadas dentro de determinadas condiçóes, em troca de pagamento de royalties (T'HOEN, 2009).

A proposta foi vista como uma possibilidade de promover mais concorrência entre os produtores que viessem buscar as licenças e também de acelerar o desenvolvimento de combinaçôes em doses fixas, cujos medicamentos isolados pertencessem a diferentes titulares, e de formas farmacêuticas pediátricas. A gestão de licenças a partir de um único local possibilita que custos de transação e negociaçôes caso a caso sejam minimizados (T'HOEN, 2009). 
Em 2010, a Unitaid decidiu investir nesta abordagem de gestão da propriedade intelectual e no mesmo ano estabeleceu o Medicines Patent Pool (MPP) (BERMUDEZ; T'HOEN, 2010), tendo como atividade central negociar e obter licenças voluntárias das empresas farmacêuticas multinacionais, buscando uma orientação para a saúde pública.

A primeira licença do MPP com uma empresa farmacêutica multinacional foi obtida em julho de 2011 com a Gilead (MEDICINES PATENT POOL, 2011) para o TDF. Esta licença gerou diferentes reaçóes críticas por parte de grupos da sociedade civil em todo o mundo, especialmente grupos e pessoas vivendo com HIV/Aids (ITPC, 2011; IP-WATCH, 2011; KEI, 2011; BAKER, 2011). Algumas das questóes levantadas era que a licença teria o potencial de minar a utilização das salvaguardas de proteção da saúde pública do Trips e que havia restrição no escopo geográfico de países beneficiados e nas condições de atuação das empresas licenciadas (WHO, 2012).

Uma das condiçôes previstas nessas licenças foi a restrição do escopo geográfico para exportação. Ou seja, quando essas empresas indianas obtiveram licenças voluntárias com a Gilead, elas se comprometeram a não exportar para alguns países em desenvolvimento, entre os quais o Brasil. Assim, mesmo existindo alternativas internacionais de genéricos do TDF, na realidade, o único produtor do qual o Brasil poderia importar era a Cipla. Todavia, não foi localizado registro sanitário para o TDF desta empresa no Brasil (VILLARDI, 2012).

O mercado público de ARV é valioso para as empresas e é provável que esta razão seja orientadora das práticas aqui discutidas. O Quadro 1 apresenta o valor do mercado, em termos de vendas do TDF pela Gilead, tanto no mundo como no Brasil, abrangendo o período em que a empresa foi fornecedora do medicamento para o MS (2004 a 2010). 
Quadro 1. Fatia do mercado brasileiro do TDF em relação ao mercado mundial em vendas do TDF para Gilead. Brasil, 2004 a 2010

\begin{tabular}{|c|c|c|c|}
\hline Ano & $\begin{array}{c}\text { Mercado mundial } \\
\text { (vendas) do TDF pela } \\
\text { Gilead (US\$)* (A1) }\end{array}$ & $\begin{array}{c}\text { Gasto do Ministério } \\
\text { da Saúde (US\$)** } \\
(\text { A2) }\end{array}$ & $\begin{array}{c}\text { Fatia do mercado } \\
\text { brasileiro (A2/ } \\
\text { A1)*100 }\end{array}$ \\
\hline 2004 & 783.000 .000 & 15.638 .757 & $1,99 \%$ \\
\hline 2005 & 778.000 .000 & 43.922 .306 & $5,64 \%$ \\
\hline 2006 & 689.000 .000 & 33.239 .741 & $4,82 \%$ \\
\hline 2007 & 613.000 .000 & 46.088 .095 & $7,51 \%$ \\
\hline 2008 & 621.000 .000 & 40.071 .405 & $6,45 \%$ \\
\hline 2009 & 667.000 .000 & 57.218 .662 & $8,57 \%$ \\
\hline 2010 & 732.000 .000 & 68.596 .262 & $9,37 \%$ \\
\hline
\end{tabular}

Fonte: Chaves (2016), a partir de dados coletados.

${ }^{*}$ MSF, 2013. Untangling the web of antiretroviral price reduction, 16th Edition.

** Dados dos preços obtidos em $\mathrm{R} \$$ e convertidos para o dólar médio do ano segundo IPEA-data.

\section{Produção local: a PDP do tenofovir}

Em 2007, o MS publicou o planejamento federal em saúde para o período de 2008 a 2011 ("Mais Saúde-Direito de Todos"), o qual estabeleceu entre os dez eixos de intervenção - com diretrizes, medidas e metas prioritárias - o Complexo Industrial da Saúde (CIS), referente à política industrial para o setor saúde. Entre as medidas previstas constavam: investimento nos Laboratórios Farmacêuticos Oficiais (LFO) e na transferência de tecnologia de farmoquímicos (princípio ativo), incluindo a nacionalizaçáo dos ARV. Uma das justificativas para o fortalecimento do CIS foi a vulnerabilidade da política nacional decorrente da dependência de importação de tecnologias de alto valor agregado (BRASIL, 2008; CHAVES, 2016).

Uma das abordagens para produção local foi a constituição de Parcerias para Desenvolvimento Produtivo envolvendo a transferência de tecnologia do medicamento de empresas privadas para LFO.

As primeiras PDP foram anunciadas pelo MS em 2009, sendo que duas eram relacionadas ao TDF (BRASIL, 2014b). Uma PDP envolveu o LFO Fundação 
Ezequiel Dias (Funed) e as empresas privadas de capital nacional Nortec Química e Blanver Produção Farmacêutica e Adjuvantes. A segunda PDP envolveu o Laboratório Farmacêutico de Pernambuco (Lafepe) e a empresa nacional Cristália, mas não foi analisada no presente estudo. A PDP do TDF foi anterior à primeira regulamentação das próprias PDPs - a Portaria no 837/2012 (BRASIL, 2012).

O laboratório Funed foi o receptor da tecnologia; a Blanver, o transferidor da tecnologia do produto final; e a Nortec, a produtora do insumo farmacêutico ativo (IFA). A empresa Gilead não foi envolvida no processo de TT devido ao não reconhecimento de sua patente no Brasil, conforme examinado na seçâo anterior.

O TDF foi o primeiro produto PDP disponibilizado e vendido para o MS em maio de 2011. Entre o período do anúncio e a primeira entrega do produto - de 2009 a 2011 -, foi feito o desenvolvimento do medicamento pela empresa Blanver. A partir da Portaria no 2.531/2014, que regulamenta as PDP e substituiu a Portaria no 837/2012, nesse período ocorreu o equivalente às fases I e II da PDP.

O desenvolvimento do medicamento pela empresa Blanver contou com a experiência da Funed para elaboração do dossiê para solicitação do registro sanitário na Anvisa, já que este produto representou para a empresa sua primeira solicitação de registro sanitário (representante empresa nacional privada\#1). Os registros foram concedidos pela Anvisa à Funed e à Blanver, respectivamente, em dezembro de 2010 e março de 2011 (VILLARDI, 2012).

O início do processo de transferência de tecnologia da Blanver para a Funed ocorreu em 2011. Embora o contrato tivesse validade de 12 meses (cláusula 4a), previa um cronograma para a transferência de três anos (termo de referência $\mathrm{N}^{\circ} 01$ ), ou seja, até dezembro de 2013. Isso significa, portanto, que o produto fornecido ao MS em maio de 2011 foi de registro sanitário da Funed com a produção feita pela Blanver, no marco do acordo de transferência de tecnologia.

O contrato (cláusula $2^{\mathrm{a}}$ ) previa valores de pagamento para três anos, apesar de a vigência ser de 12 meses, e relacionava um quantitativo de produção. A diferença entre o preço do tenofovir praticado pela Funed ao MS e o preço do comprimido no contexto da transferência de tecnologia e produçáo foi estimado. $\mathrm{O}$ valor do contrato da Funed com o consórcio Blanver-Nortec para a transferência de tecnologia e fornecimento do produto ao MS foi de $\mathrm{R} \$ 103.680 .000$ para 28.000.000 de 
comprimidos, equivalendo a um preço unitário de $\mathrm{R} \$ 3,6$. O valor pago pelo MS à Funed foi de $\mathrm{R} \$ 4,02$ por unidade. Isso significa que Laboratório Público acrescentou $\mathrm{R} \$ 0,42$ por unidade farmacêutica além do valor repassado aos parceiros privados do consórcio, totalizando R \$ 12.096.000 no primeiro ano.

Conforme foi, posteriormente, estabelecido pela Portaria no 837/2012, durante a vigência da PDP era admitida a previsão da alguma margem sobre o preço do produto: III - quanto ao processo das PDP:

$[\ldots]$

$\$ 1^{\circ}$ Excepcionalmente, no regime das PDP os preços podem incluir uma margem, sobre os custos negociados, para a integração das tecnologias estratégicas para o SUS, desde que justificados pelo aporte tecnológico associado à internalizado da produção e pela relevância do bem ou produto para saúde pública.

Esta prerrogativa pode explicar a diferença entre o preço praticado pelo laboratório oficial ao MS e aquele praticado no marco do consórcio com as empresas privadas envolvidas na PDP. O contrato de fornecimento do TDF, a partir de 2011, pode ser considerado o início do processo de TT para o LFO, que, posteriormente, veio a ser chamado de fase III da PDP (BRASIL, 2014b).

O desenvolvimento do IFA também não envolveu transferência de tecnologia da Gilead. No entanto, as duas empresas nacionais envolvidas nas PDPs do TDF, Nortec e Cristália, haviam trabalhado em colaboração em um projeto financiado pela Finep, no início dos anos 2000, no desenvolvimento do IFA do TDF. O marco da PDP significou um novo desafio a essas empresas em funçáo do aumento da escala de produção e da necessidade de cumprimento das exigências regulatórias (VERAS, 2014). Em 2016, essas PDPs chegaram à fase IV, referente à internalização da tecnologia nos laboratórios oficiais (BRASIL, 2017).

A última análise foi a comparação do preço do produto PDP com o preço de alternativa genérica disponível no mercado internacional. O Gráfico 1 mostra a comparação dos preços convertidos pelo dólar médio do ano e os preços ofertados pela empresa Cipla. 
Gráfico 1. Evoluçẫo do preço do TDF 300 mg praticado no Brasil (US\$) e comparação com o preço da versão genérica da Cipla (US\$). Brasil, 2003 a 2013

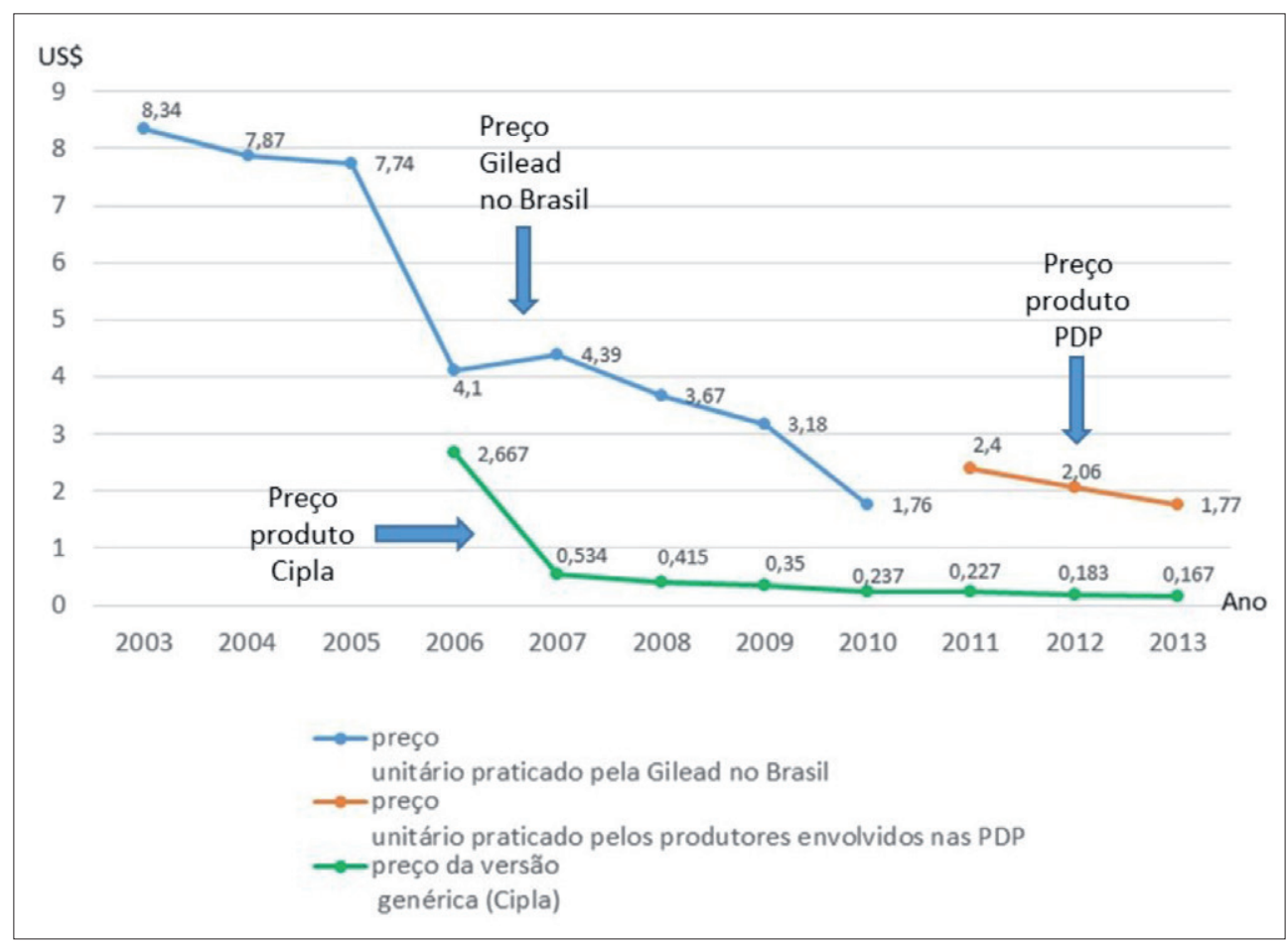

Fonte: Chaves (2016), a partir dos dados fornecidos pelo MS e consulta à base de preços dos MSF.

O gráfico 1 aponta para uma diferença de cerca de dez vezes entre o preço do produto nacional e o preço do genérico ofertado pela Cipla. A empresa, como visto, não fez acordos de licença voluntária com a Gilead, que pudessem restringir a oferta ao Brasil. Entretanto, até 2012 náo havia registro sanitário da empresa para este medicamento no País, o que na prática significava a não oferta real do medicamento a um menor preço no país.

Conforme se descreve na próxima seção, quando o preço foi analisado em reais (R\$), com valores ajustados pelo IPCA, há uma redução sistemática. No entanto, na análise do preço em dólar, o produto nacional sinalizou um aumento em relação ao preço praticado pela Gilead, em 2010, porque houve queda do dólar entre 2010 e 2011 (o câmbio passou de $\mathrm{R} \$ 1,76$ para $\mathrm{R} \$ 1,67$ ).

A partir de 2009, o produto da Cipla passou a ser pré-qualificado pela OMS (WHO, 2012), o que poderia ter facilitado a compra por agências internacionais (por exemplo, 
OPAS ou Unicef) e, consequentemente, a sua entrada no país apesar da ausência de registro sanitário. De qualquer forma, o fato de que a Cipla tenha sistematicamente reduzido o preço do produto ao longo dos anos deve servir de referência aos preços praticados nacionalmente mesmo no âmbito da política industrial, especialmente quando o produto em questão refletir um aumento considerável do volume adquirido e do gasto do MS, como foi o caso do tenofovir no período analisado.

Estudo recente (VILLARDI; SCOPEL, 2017) compara o custo de produção considerando o preço do IFA na PDP e no mercado internacional, e sugere que o custo do comprimido de TDF na PDP foi 2,3 vezes maior do que se tivesse sido utilizado o IFA internacional. Uma das possibilidades de ampliação da escala de compra seria a comercialização no mercado internacional, principalmente pelos parceiros privados. Isso poderia contribuir para a redução dos preços ofertados no Brasil. Os produtores privados nacionais (farmacêuticos e farmoquímicos) têm a oportunidade de exportar para os países que não tenham patente para o tenofovir e que tenham sido excluídos da chance de obter versóes genéricas pela licença do MPP ou que tenham patente e venham a emitir uma licença compulsória.

\section{Efeitos das iniciativas na evoluçáo do gasto e do preço do TDF}

Os gráficos 2, 3 e 4 apresentam, respectivamente, a evolução do preço, do gasto do MS e do volume de compra do TDF no Brasil para o período de 2003 a 2013.

Quando ajustados ao IPCA, é possível observar uma redução sistemática do preço do tenofovir entre 2003 e 2013, incluindo durante a vigência da PDP (2011 a 2013) (gráfico 2). Entre 2005 e 2006, observa-se uma redução do preço em 53,7\% (gráfico 2) e uma diminuição do gasto em 2006 (gráfico 3), apesar do aumento do volume da compra (gráfico 4). Em 2008, nota-se também uma redução do gasto total com a compra de tenofovir (gráfico 3), apesar do aumento do volume (gráfico 4).

No final da década de 1990, o Ministério da Saúde iniciou processos de negociação de preços de ARV sob monopólio com as empresas farmacêuticas multinacionais, utilizando uma série de argumentos que pudessem aumentar o seu poder de barganha, dentre os quais a ameaça de emissão de licença compulsória. Ao ser incorporado em 2003, o TDF passou a fazer parte do elenco de produtos que comprometiam o orçamento ARV, tendo sido por isso objeto de negociaçóes de preços ao longo dos anos (BERMUDEZ et al., 2004; BARROS; CASTRO, 2014; CHAVES, 2016). 
Em 2009, ocorreram dois movimentos importantes relacionados à situação de monopólio do tenofovir no Brasil: o pedido de patente foi definitivamente indeferido pelo INPI e o MS anunciou a PDP do medicamento para produçâo local. No ano seguinte, observa-se uma redução de $40 \%$ no preço unitário de venda do produto da Gilead, que passou de $\mathrm{R} \$$ 8,03 por comprimido para $\mathrm{R} \$ 4,81$ (gráfico 2). Importante notar que, apesar da redução do preço, no ano de 2010 houve aumento de $41 \%$ no volume de compra do MS (gráfico 4) e dos gastos (em cerca de 20\%) (gráfico 3).

O gasto total de 2009 e 2010 se manteve constante, em torno de R $\$ 144$ milhóes, apesar do aumento do volume. Este resultado evidencia dois efeitos: fim da expectativa de monopólio e anúncio de entrada de um novo produtor por meio da PDP. Dessa forma, o MS pode ampliar o volume de compras com a manutenção dos gastos totais para este medicamento.

No período de 2006 a 2010, a Gilead foi o único fornecedor do TDF ao Ministério da Saúde, muito embora já existissem no mercado internacional versôes genéricas. Considerando a possibilidade de importação da versão genérica do medicamento pela empresa Cipla, a estimativa do gasto adicional do Ministério da Saúde foi de cerca de US\$ 200 milhóes (Quadro 2).

Gráfico 2. Evolução do preço unitário do Tenofovir $300 \mathrm{mg}$ e iniciativas dos diferentes sujeitos relacionadas. Brasil, 2003 a 2013

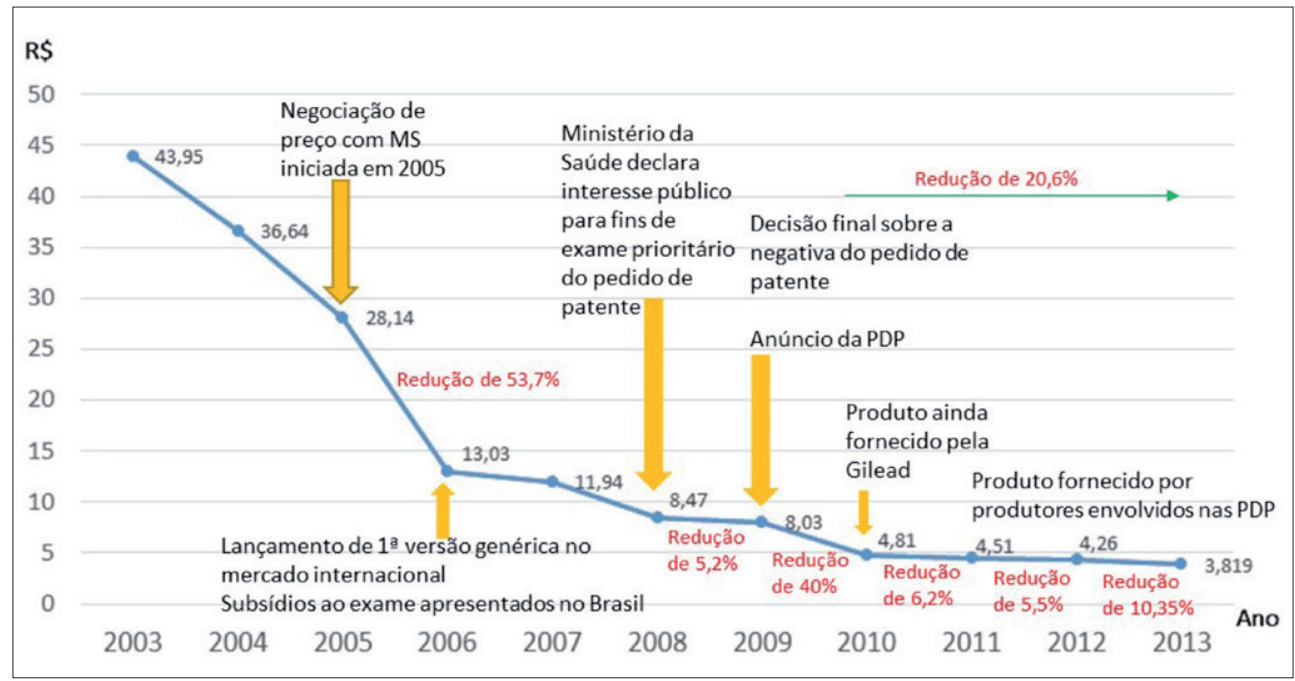

Fonte: Chaves (2016), a partir de dados preços, fornecidos pelo MS e corrigidos pelo IPCA de 2013, e mapeamento das iniciativas. 
Gráfico 3. Evolução do gasto* com Tenofovir 300 mg pelo Ministério da Saúde. Brasil, 2003 a 2013

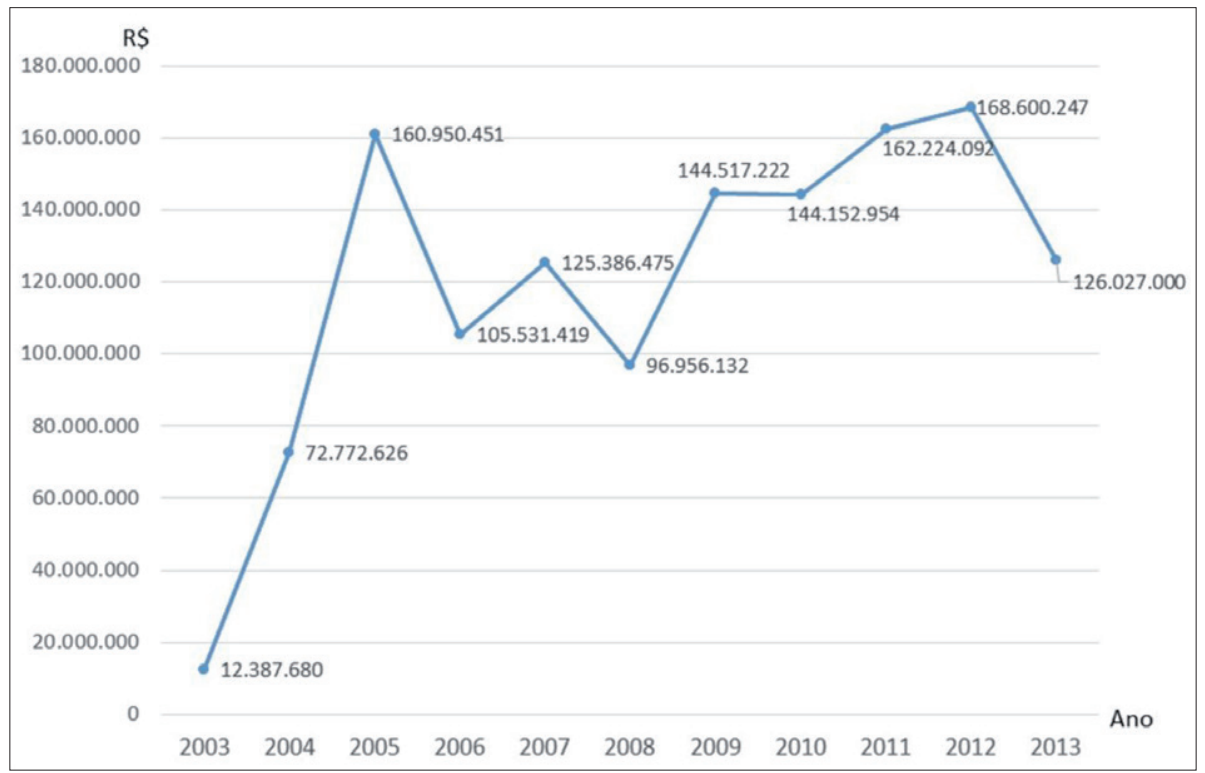

Fonte: Chaves (2016), a partir de dados fornecidos pelo Ministério da Saúde.

* Dados ajustados pelo IPCA, calculados a partir dos preços e volumes fornecidos pelo MS.

Gráfico 4. Volume de comprimidos adquiridos por ano expressos em número de tratamentos por ano*. Brasil, 2003 a 2013

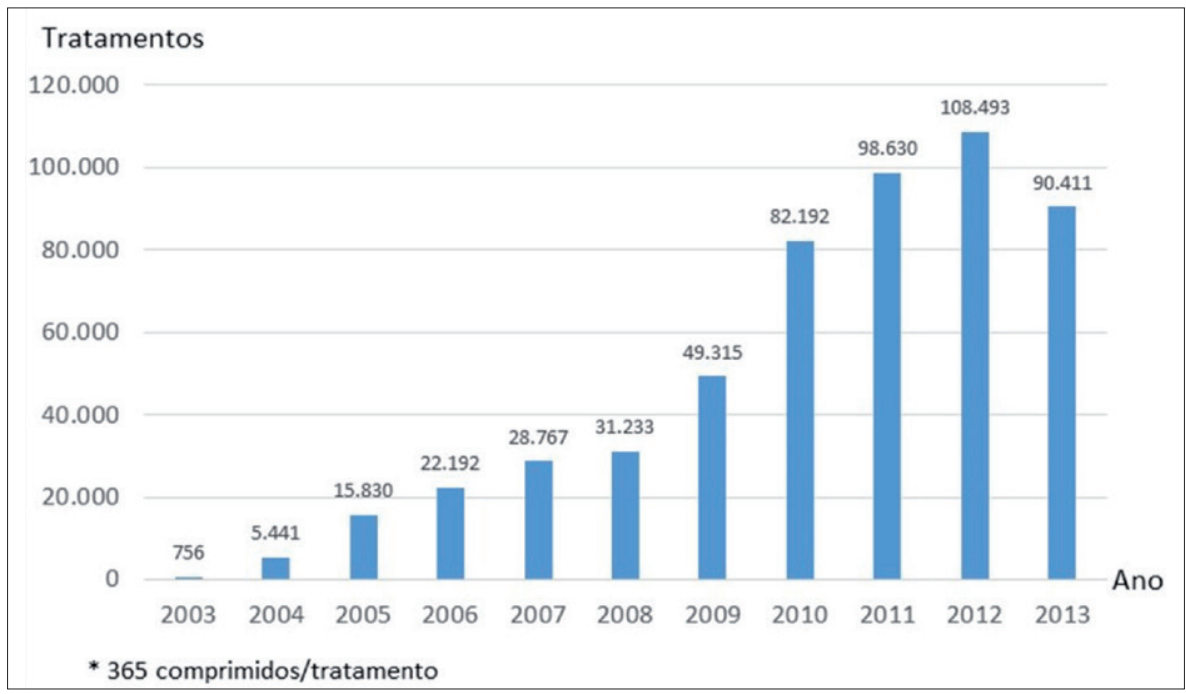

Fonte: Chaves (2016), a partir de dados fornecidos pelo Ministério da Saúde. 
Quadro 2. Diferença entre os gastos do Ministério da Saúde com TDF 300 mg com o preço praticado pela Gilead e a estimativa do gasto com o preço da versấo genérica, 2006 a 2010

\begin{tabular}{|l|l|l|l|l|l|l|}
\hline & 2006 & 2007 & 2008 & 2009 & 2010 & $\begin{array}{l}\text { Total } \\
(2006-2010)\end{array}$ \\
\hline $\begin{array}{l}\text { Gasto do } \\
\text { Ministério da } \\
\text { Saúde com o TDF } \\
300 \text { mg (preço } \\
\text { Gilead US\$) (A) }\end{array}$ & $33.241 .268,4$ & 46.085 .729 & $40.084 .514,7$ & $57.235 .853,8$ & $68.607 .654,5$ & $245.255 .020,4$ \\
\hline $\begin{array}{l}\text { Gasto estimado } \\
\text { com TDF 300 } \\
\text { mg (preço Cipla, } \\
\text { US\$) (B) }\end{array}$ & 21.602 .700 & 5.607 .000 & 4.731 .000 & 6.300 .000 & 7.110 .000 & 45.350 .700 \\
\hline $\begin{array}{l}\text { Estimativa do } \\
\text { gasto adicional } \\
\text { pago pelo } \\
\begin{array}{l}\text { Ministério da } \\
\text { Saúde pelo preço } \\
\text { de monopólio } \\
\text { (A-B) }\end{array}\end{array}$ & $11.638 .568,4$ & 40.478 .729 & $35.353 .514,7$ & $50.935 .853,8$ & $61.497 .954,5$ & $199.904 .620,4$ \\
\hline
\end{tabular}

Fonte: Preços da Cipla do MSF Untangling the Web of Price Reductions. Estimativas de gasto baseadas nos preços multiplicados pelas unidades farmacêuticas adquiridas pelo Ministério da Saúde no período.

Em relação ao preço praticado no contexto da PDP, o preço inicialmente apresentado no projeto executivo para a implementação da PDP do tenofovir foi cerca de R \$ 5,32. No entanto, quando saiu o registro do produto nacional, a Gilead reduziu o preço consideravelmente e o laboratório oficial teve que acompanhar esta redução no âmbito da PDP (entrevista empresa nacional privada\#1).

A questão do preço do produto PDP é um dos componentes levados em conta no processo, muito embora tenha sido mencionado por entrevistados como não sendo a principal finalidade da PDP. No entanto, mesmo não sendo a principal finalidade, o MS exigiu desde o início do projeto de PDP que o preço fosse inferior ao preço do produto previamente adquirido pelo SUS (gestores públicos do Ministério da Saúde \#2, \#3). Este parâmetro veio a ser incorporado na Portaria no 837/2012 e, posteriormente, na Portaria $n^{\circ} 2.531 / 2014$.

Dessa forma, o caso demonstra dois efeitos reguladores do preço: de um lado, a empresa multinacional reduziu o preço do produto de referência em resposta à negativa da patente e à ameaça da entrada de novo concorrente no mercado nacional; 
de outro, os produtores nacionais, que passariam a se beneficiar da exclusividade do mercado público no contexto da PDP, tiveram que reduzir o preço de suas ofertas iniciais ao MS a partir da redução do preço pela empresa multinacional.

Em relação aos gastos totais, com a compra de tenofovir no período da aquisição PDP (Gráfico 3), observa-se que de 2010 a 2011 o aumento do gasto foi de 11,2\% para um aumento do volume de $16,7 \%$. Em 2012, o aumento do volume foi de $9,1 \%$, acompanhado de um acréscimo de 3,8\% do gasto total em relação a 2011 . Em 2013, houve uma redução de $16,7 \%$ do volume, acompanhada de uma diminuição de $25,26 \%$ do gasto do total. Estes dados demonstram que houve redução do preço unitário do produto PDP no período coberto pela PDP.

\section{Considerações finais}

As iniciativas de superação da barreira patentária implementadas em 2005 e 2006, por meio dos subsídios ao exame, surtiram efeito no médio prazo ao contribuir para a decisão do INPI pelo indeferimento do pedido de patente em 2009. Um reflexo deste movimento foi a redução do preço em 2010.

Este estudo demonstrou o impacto do monopólio envolvendo um medicamento com pedidos de patente pendentes nos gastos adicionais do Ministério da Saúde. Se, por um lado, a expectativa do direito prevista na legislação de propriedade industrial assegura indenização retroativa no caso de o pedido vir a ser concedido; por outro, há que se buscar caminhos e interpretaçóes legais que possibilitem a compensação do preço de monopólio pago a um medicamento cujo pedido de patente venha a ser negado, ou seja, a compensação de um monopólio indevido.

Os diferentes esforços de superação da barreira patentária no país refletiram uma oportunidade para produção local no marco da nova política industrial estabelecida a partir de 2008. O Brasil passou da importação do produto de referência da Gilead para a compra do produto PDP envolvendo produtores públicos e privados nacionais. Este caso ilustra o efeito da concorrência em duas situaçôes específicas: primeiro, pela redução significativa do preço ofertado pela Gilead após a negativa da patente e o anúncio da PDP; e segundo, pelo estabelecimento do preço de referência para o produto PDP a partir de 2011.

Cabe ressaltar que os esforços de superação da barreira patentária para promover um ambiente concorrencial foram substituídos pelo monopólio da PDP sem 
considerar alternativas simultâneas. A análise do preço, volume e gasto com o medicamento no período estudado sugere que as diferentes iniciativas envolvendo enfrentamento da barreira patentária e produção local resultaram em redução do preço, com aumento do volume da compra e relativa estabilidade do gasto.

Dada a complexidade da regulação de preços de produtos sob monopólio, o presente estudo mostrou aspectos da dinâmica do mercado pouco aprofundados na literatura da saúde coletiva e, ao mesmo tempo, essenciais para a garantia da sustentabilidade e do acesso universal a medicamentos no âmbito do Sistema Único de Saúde. ${ }^{3}$

\section{Agradecimentos}

O presente estudo é um dos resultados da tese de doutorado intitulada "Interfaces entre a produçáo local e o acesso a medicamentos no contexto do Acordo TRIPS da Organização Mundial do Comércio” realizada pela primeira autora e orientada pelas outras autoras. Ela foi defendida em maio de 2015 pelo Programa de PósGraduação em Saúde Pública da Escola Nacional de Saúde Pública Sergio Arouca, Fundação Oswaldo Cruz.

A pesquisa contou com financiamento do Programa de Pós-Graduação em Saúde Pública da ENSP/Fiocruz e do CNPq (Edital MCTI/CNPq/CT-Saúde/MS/ SCTIE/Decit N o 41/2013 referente à "Rede Nacional de Pesquisas sobre Política de Saúde: Conhecimento para Efetivação do Direito Universal à Saúde”).

\section{Referências}

ABREU, J. C. Prospecção tecnológica aplicada na otimização da concessão de patentes no Brasil: estudo de caso em patentes de medicamentos imunossupressores. 2017. Tese (Doutorado) Universidade Federal do Rio de Janeiro, Rio de Janeiro, 2017.

ACCESS CAMPAIGN/MEDECINS SANS FRONTIÈRES. Untangling the web of price reductions: a pricing guide for the purchase of ARVs for developing countries. Genebra: MSF, 2014. Disponível em: <https://www.msfaccess.org/content/untangling-web-antiretroviral-pricereductions $>$. Acesso em: 15 out. 2014.

. Untangling the web of price reductions: a pricing guide for the purchase of $A R V$ for developing countries. Genebra: MSF, 2007.

. Untangling the Web of Antiretroviral Price Reductions. Genebra: MSF, 2013. Disponível em: <AIDS_Report_UTW16_ENG_2013.pdf>. Acesso em: 21 out. 2014. 
. Patent Opposition Database. Disponível em: < <https://www.patentoppositions.org/en/ drugs/tenofovir-disoproxil-fumarate.> Acesso em 05/03/2018

AGÊNCIA FIOCRUZ DE NOTÍCIAS. Parcerias público-privadas viabilizam produção nacional de 24 fármacos. 14 abr. 2009.

AGÊNCIA NACIONAL DE VIGILÂNCIA SANITÁRIA (ANVISA). Resolução CMED N 2, de 5 de março de 2004. Ficam aprovados, na forma do Anexo a esta Resolução, os critérios para definição de preços de produtos novos e novas apresentaçóes de que trata o art. $7^{\circ} \mathrm{da}$ Lei $\mathrm{n}^{\circ}$ 10.742, de 6 de outubro de 2003.

BAKER, B. Inside view: Corporate self-interest and strategic choices: Gilead licenses to Medicines Patent PoolIP-watch. 21 jul. 2011. Disponível em: <http://www.ip-watch.org/2011/07/21/ corporate-self-interest-and-strategic-choices-gilead-licenses-to-medicines-patent-pool/>. Acesso em: 26 jul. 2014.

BARROSO, W. Contribuição ao estudo do subsídio ao exame de pedido de patente no Brasil. In: Aids e Saúde Pública: contribuiçóes à reflexão sobre uma nova economia política do medicamento no Brasil. Rio de Janeiro: EdUERJ, 2010.

. Procedimento de oposição: o caso Tenofovir. In: Propriedade intelectual e políticas públicas para o accesso aos antirretrovirais nos paises do Sul. Rio de Janeiro: ANRS: E-papers, 2013.

BARROS e CASTRO MT de. Licenciamento Compulsória no Brasil: instituiçóes e políticas [Tese de doutorado]. Rio de Janeiro: Instituto de Economia, Universidade Federal do Rio de Janeiro; 2014.

BERMUDEZ, J.; T'HOEN, E. The UNITAID patent pool initiative: bringing patents together for the common good. The open AIDS journal, v. 4, 2010, p. 37.

BRASIL. Lei no 9.279, de 14 de maio de 1996. Regula direitos e obrigaçôes relativos à propriedade industrial. 1996.

. CONSELHO NACIONAL DE SAÚDE. Resolução $N^{\circ}$ 338, de 6 de maio de 2004. Aprova a Política nacional de Assistência Farmacêutica. 2004. Disponível em: <http://bvsms. saude.gov.br/bvs/saudelegis/cns/2004/res0338_06_05_2004.html>.

. MINISTÉRIO DA SAÚDE. Portaria $M S / G M n^{\circ}$ 681. Declara de interesse público o medicamento anti-retroviral Tenofovir para fins de exame prioritário de pedido de patente junto ao Instituto Nacional de Propriedade Industrial - INPI. Abril de 2008.

. MINISTERIO DA SAÚDE, Secretaria de Ciência, Tecnologia e Insumos Estratégicos. Departamento de Assistência Farmacêutica e Insumos Estratégicos. Serviços farmacêuticos na atenção básica à saúde. - Brasília : Ministério da Saúde, 2014a. 108 p. : il. - (Cuidado farmacêutico na atenção básica ; caderno 1) 
_b. MINISTERIO DA SAÚDE, Gabinete do Ministro. Redefine as diretrizes e os critérios para a definição da lista de produtos estratégicos para o Sistema Único de Saúde (SUS) e o estabelecimento das Parcerias para o Desenvolvimento Produtivo (PDP) e disciplina os respectivos processos de submissão, instrução, decisão, transferência e absorção de tecnologia, aquisição de produtos estratégicos para o SUS no âmbito das PDP e o respectivo monitoramento e avaliação. Brasilia: Ministerio da Saúde. 12 de novembro de 2014 b.

MINISTERIO DA SAÚDE, Gabinete do Ministro. Portaria no 837/GM/MS . Define as diretrizes e os critérios para o estabelecimento das Parcerias para o Desenvolvimento Produtivo (PDP). Brasilia, 18 de abril de 2012. Disponivel em: <http://bvsms.saude.gov.br/bvs/ saudelegis/gm/2012/prt0837_18_04_2012.html>

- Propostas de Projetos de Parcerias para o Desenvolvimento Produtivo (PDP) aprovadas até dezembro de 2013. Disponível em: <http://portalsaude.saude.gov.br/images/pdf/2015/ janeiro/08/Propostas-de-Projetos-de-Parcerias-para-o-Desenvolvimento-Produtivo--PDP-aprovadas-at---dezembro-de-2013---08-01-14.pdf>. Acesso em: 7 mar. 2016b.

- Gabinete do Ministro. Portaria MS/GM n 3916, de 30 de outubro de 1998. Aprova a Política Nacional de Medicamentos. Brasília, DF: Diário Oficial da República Federativa do Brasil, 10 nov. 1998.

BRASIL. Ministério da Saúde. Fase IV - Internalização da Tecnologia. Lista atualizada em 7/5/2017. Disponível em: <http://portalarquivos.saude.gov.br/images/pdf/2017/maio/12/FaseIV---atualizada-em-09.05.2017.pdf>. Acesso em: 25/6/2017.

. Presidência da República, Casa Civil. Lei no 8080, de 19 de setembro de 1990. Dispóe sobre as condiçóes para a promoção, proteção e recuperação da saúde, a organização e o funcionamento dos serviços correspondentes e dá outras providências. 1990.

CÂMARA DOS DEPUTADOS, BRASIL. A revisão da Lei de Patentes: inovação em prol da competitividade nacional. [s.l.]: Ediçôes Câmara, 2013.

CHAVES, G.C. et al. Strategies for price reduction of HIV medicines under a monopoly situation in Brazil. Revista de Saúde Pública, v. 49, 2015.

CHAVES, G.C.; VIEIRA, M.F.; REIS, R. Acesso a Medicamentos e Propriedade Intelectual no Brasil: reflexões e estratégias da sociedade civil. Sur-Revista Internacional de Direitos Humanos, v. 8, p. 171-197, 2008.

CHAVES, G.C.; REIS, R. Health, Intellectual Property and Innovation Policy: a case study of Brazil. In: Pharmaceutical Innovation, Incremental Patenting and Compulsory Licensing. Genebra: South Centre, 2013, p. 337.

CHAVES, G.C. Interfaces entre produçâo local e acesso a medicamentos no contexto do Acordo TRIPS da Organização Mundial do Comércio. 2016. Tese (Doutorado) - ENSP/Fiocruz, Rio de Janeiro, 2016. 
CONSELHO NACIONAL DE SAÚDE. Resolução 352, agosto de 2005.

CONSULTATIVE EXPERT WORKING GROUP ON RESEARCH AND DEVELOPMENT: FINANCING AND COORDINATION. Research and Development to Meet Health Needs in Developing Countries: Strengthening Global Financing and Coordination. Genebra: World Health Organization. Abril 2012. Disponível em: <http://www.who.int/phi/ CEWG_Report_5_April_2012.pdf>. Acesso em: 29 abr. 2013.

FLYNN, M.B. Pharmaceutical Governance in Brazil: Globalization, Institutions and AIDS. Tese (Doutorado em Filosofia) - University of Texas, Austin, 2010.

FONSECA, E.M. da; COSTA, N. do R. Federalism, the Economic-Industrial Health Care Complex and High-Cost Pharmaceutical Assistance in Brazil. Ciência \& Saúde Coletiva, v. 20, n. 4, p. 1.165-1.176, abr. 2015.

GRANGEIRO, A. et al. Sustentabilidade da política de acesso a medicamentos anti-retrovirais no Brasil. Revista de Saúde Pública de São Paulo, n. 40 (supl.), p. 60-9, 2006.

HASENCLEVER, L. et al. O instituto de patentes Pipeline e o acesso a medicamentos: aspectos econômicos e jurídicos deletérios à economia da saúde. Revista de Direito Sanitário, v. 11, n. 2 , p. $164-188,2010$.

INPI. Busca-patentes. Disponível em: <http://www.inpi.gov.br/portal/artigo/busca_patentes>. Acesso em: 25 nov. 2017.

INSTITUTO DE PESQUISA ECONÔMICA APLICADA. IPEA data. Disponível em: <http://www.ipeadata.gov.br/>. Acesso em: 24 abr. 2013.

INTERNATIONAL TREATMENT PREPAREDNESS COALITION (ITPC). Concerns about the process, principles of Medicines Patent Pool and the licence. Disponível em: <http://www. petitionbuzz.com/petitions/mppunitaid>. Acesso em: 11 maio. 2014.

IP-WATCH. Brief: Medicines Patent Pool Responds To Critics Of Gilead Licence. 11 ago. 2011.

KAPCZYNSKI, A.; PARK, C.; SAMPAT, B. Polymorphs and Prodrugs and Salts (Oh My!): An Empirical Analysis of "Secondary" Pharmaceutical Patents. PLoS ONE. Mintzes B, editor, 5;7(12):e49470, Dec. 2012.

KNOWLEDGE ECOLOGY INTERNATIONAL. KEI comment on the Medicines Patent Pool license with Gilead. Washington: KEI, 12 jul. 2011. Disponível em: <http://www.keionline.org/ node/1184>. Acesso em: 10 mar. 2014.

MEDICINES PATENT POOL. Medicines Patent Pool Announces First Licensing Agreement with a Pharmaceutical Company. 12 jul. 2011.

MEINERS, C. M. M. de A. Pharmaceutical technology incorporation and affordability of HIVIAIDS treatment in developing countries: an analysis of the Brazilian response. Rio de Janeiro;Marseille: Universidade Federal do Rio de Janeiro:Aix-Marseille University, 2012. 
MINISTÉRIO DA SAÚDE. Secretaria Executiva. Mais saúde : direito de todos: 2008 - 2011: Série C. Projetos, Programas e Relatórios. Brasília: Ministério da Saúde, 2008. Disponível em: <http://bvsms.saude.gov.br/bvs/publicacoes/mais_saude_direito_todos_2ed.pdf>. Acesso em: 16 mar. 2015.

NUNN, A. S. et al. Evolution of Antiretroviral Drug Costs in Brazil in the Context of Free and Universal Access to AIDS Treatment. PLoS Medicine, v. 4, n. 11, p. e305, 2007.

OliveirA, M. A.; BermudEZ, J. A. Z.; CHAVES, G. C.; VELÁSQUEZ, G. Has the implementation of the TRIPS Agreement in Latin America and the Caribbean produced intellectual property legislation that favours public health? Bulletin of the World Health Organization, v. 82, n. 11p. 815-21, 2004.

PUBLIC PATENT FOUDANTION. PUBPAT takes on key HIVIAIDS drug patents: Non-Profit Submits Prior Art to U.S. Patent Office and Asks For Revocation of Four Gilead Sciences Patents. 2007. Disponível em: <http://www.pubpat.org/gileadhivfiled.htm>. Acesso em: 7 mar. 2016.

REIS, R. Panorama Patentário dos Medicamentos Antirretrovirais no Brasil. 2012. Tese (Doutorado em Políticas Públicas, Estratégias e Desenvolvimento) - Universidade Federal do Rio de Janeiro, Rio de Janeiro, 2012.

REIS, R.; VIEIRA, M.; CHAVES, G. Acesso a medicamentos e propriedade intelectual no Brasil: a experiência da sociedade civil. In: Direitos de Propriedade Intelectual e acesso aos Antirretrovirais: resistência da sociedade civil no Sul Global. Rio de Janeiro: ABIA, 2009, p. 12-58.

SCHEFFER, M. C. Aids, tecnologia e acesso sustentável a medicamentos: a incorporação dos anti-retrovirais no Sistema Único de Saúde. Tese (Doutorado) - Faculdade de Medicina, Departamento de Medicina Preventiva, Universidade de São Paulo, São Paulo, 2008.

SCHEFFER, M. C.; SALAZAR, A.; GROU, K. O remédio via Justiça - Um estudo sobre o acesso a novos medicamentos e exames em HIVIAids no Brasil por meio de açôes judiciais. Ministério da Saúde, 2005.

SCOPEL, C. T. ; Gabriela, C.C. Iniciativas de enfrentamento da barreira patentária e a relaçáo com o preço de medicamentos adquiridos pelo Sistema Único de Saúde. Cadernos de Saúde Pública (ENSP. Impresso), v. 32, p. 1-12, 2016.

SILVA, R. I. da. Indústria Farmacêutica Brasileira: Estrutura e a Questão dos Preços de Transferência. 1999. Tese (Doutorado) - Universidade Federal do Rio de Janeiro/Escola de Química, Rio de Janeiro, 1999.

SOOD, N. et al. The Effect Of Regulation On Pharmaceutical Revenues: Experience In Nineteen Countries. Health Affairs, v. 28, n. 1, p. w125-w137, 1 jan. 2009.

T' HOEN, E. The global politics of pharmaceutical monopoly power: drug patents, access, innovation and the application of the WTO Doha Declaration on TRIPS and public health. [s.1.]: AMB Publishers, 2009. 
VERAS, J. Making Tenofovir Accessible In The Brazilian Public Health System: Patent Conflicts And Generic Production: Making Tenofovir Accessible. Developing World Bioethics, v. 14, n. 2, p. 92-100, ago. 2014.

VIEIRA, F. S. Gasto do Ministério da Saúde com medicamentos: tendência dos programas de 2002 a 2007. Rev. Saúde Pública, v. 43, n. 4, p. 674-81, 2009.

VILLARDI, P. Panorama do status patentário e registro sanitário dos medicamentos antiretrovirais no Brasil - implicaçóes para o acesso e a política industrial no Brasil. Associação Brasileira Interdisciplinar de Aids, 2012. Disponível em: <http://www.deolhonaspatentes.org.br/media/file/ Publica\%C3\%A7\%C3\%B5es/Publica\%C3\%A7\%C3\%A3o\%20Pedro_Final_23OUT.pdf>.

VILLARDI, P.; SCOPEL, C.T. A luta pelo tenofovir: vitórias e desafios futuros. In: Políticas de produçấo local de medicamentos no Brasil: elementos para o debate. Rio de Janeiro: ABIA,p. 131-82, 2017.

WORLD HEALTH ORGANIZATION (WHO). Essential Medicines and Health Products - Prequalification of Medicines. 2012. Disponível em: <https:/extranet.who.int/prequal>. Acesso em: 20 jan. 2017.

\section{Notas}

${ }^{1}$ Trade Related Aspects of Intellectual Property Rights ou Acordo sobre os Direitos de Propriedade Intelectual relacionados ao Comércio.

${ }^{2}$ Cálculo do custo de um tratamento: 1 comprimido/dia x 365 dias x preço unitário do comprimido.

${ }^{3} \mathrm{G}$. C. Chaves coletou e analisou os dados e redigiu a primeira versão do manuscrito; participou da concepçáo do estudo e aprovação da versão final. L. Haenclever e M. A. Oliveira participaram igualmente da concepção do estudo e aprovaram a versão final. 


\section{Abstract}

Price reduction of monopoly medicine in the Unified Health System: the case of Tenofovir This study aimed to analyze the evolution of the price of tenofovir (TDF) in Brazil considering the different initiatives for its reduction. The selection criteria for the case were: to have been subject to at least one strategy to overcome patent barrier, which in the case was the support to examination of the patent application (patent opposition); and have been subject of a local production Partnership for Productive Development (PDP). The main results suggest that patent opposition presented in 2005 and 2006 contributed to the decision to reject the patent application in 2009. Brazil is estimated to have paid around US\$200 million more for the monopoly due to the patent pending application period. There was a reduction in the price of TDF between 2003 and 2013, including during the PDP (2011 to 2013). In 2010, after the PDP announcement, there was an additional $40 \%$ decrease in the price of the TDF offered by Gilead, which reflected in the price offered by the PDP. However, the price paid in Brazil for the national product was about ten times higher than the generic offered internationally.

> Keywords: drug price; HIVIAIDS; patents; generic medicines. 